\title{
Resistance to Electronic Medical Records (EMRs): A Barrier to Improved Quality of Care
}

\author{
David B. Meinert \\ Southwest Missouri State University \\ Springfield, Missouri USA
}

\author{
davidmeinert@smsu.edu
}

\begin{abstract}
While most industries have aggressively leveraged information technology (IT) to improve quality and reduce costs the healthcare sector has lagged behind. Electronic Medical Records (EMRs) hold great promise for improving quality of care yet widespread adoption is lacking. Physician acceptance is critical to widespread adoption of ambulatory EMRs, yet there is little independent research on physician perceptions. This paper attempts to address this void by reporting the results of a study of physician perceptions related to EMRs in a large, multi-specialty clinic. Physician perceptions of select EMR functions and general attitudes and beliefs are reported. While the importance and anticipated utilization of EMR functions varied, nearly 80 percent of the respondents felt an EMR should be implemented. The findings have implications for both vendors attempting to design and market EMR systems and physician executives and practice managers seeking to solicit support for EMR adoption and/or develop a successful implementation strategy.
\end{abstract}

Keywords: Electronic Medical Records (EMR), Computerized Patient Records (CPR), medical informatics applications, medical record systems, physician acceptance.

\section{Introduction}

Most industries have aggressively embraced the use of information technology (IT); however, healthcare is one noticeable exception. According to Morrissey (2005, p. 42), U.S. hospitals and multiple-facility health systems are "only beginning to round out their clinical networks, but are much farther along than physician practices." While many inpatient or hospital facilities have migrated from paper charts to electronic records little progress has been made in the ambulatory or outpatient setting (Versel, 2002). According to Versel (2002) the vast majority (greater than $85 \%$ ) of individual and group physician practices have been reluctant to embrace electronic medical records and continue to rely on paper charts. Paper medical records or charts are by nature data rich, but information poor as physicians and other health care providers have limited time to dig through volumes of paper to retrieve information, utilize it in decision-making and/or share it with patients. Ambulatory EMR systems hold tremendous promise for not only improving the quantity and quality of clinical data that can be recorded, but more importantly the ability

Material published as part of this journal, either on-line or in print, is copyrighted by Informing Science. Permission to make digital or paper copy of part or all of these works for personal or classroom use is granted without fee provided that the copies are not made or distributed for profit or commercial advantage AND that copies 1) bear this notice in full and 2) give the full citation on the first page. It is permissible to abstract these works so long as credit is given. To copy in all other cases or to republish or to post on a server or to redistribute to lists requires specific permission from the publisher at Publisher@InformingScience.org to access health care data to improve quality of care. Unfortunately, although a myriad of EMR applications/vendors are available, it has been estimated that it will take at least a decade for physician adoption to reach 80 percent (Versel, 2002). 
The purpose of this paper is threefold: 1) to examine why physicians have been reluctant to adopt EMRs; 2) to provide specific examples of how EMRs can improve quality of care; and 3) to report findings from a recent study focused on physician perceptions concerning EMRs. First, the paper examines the EMR literature to develop a basis for this investigation. The literature review necessarily examines findings related to EMRs, most notably that having to do with physician acceptance and EMR functionality. Second, the paper describes specific opportunities for improving quality of care via EMRs. Next, the paper presents findings from a 2003 study that offers insight into contemporary physician perceptions. Following a discussion of the findings both limitations of the study and opportunities for future research are presented.

\section{Background and Prior Research}

\section{Electronic Medical Records}

For nearly two decades both vendors and well-respected medical and health-care journals and magazines have touted EMRs as a means to dramatically improve physician efficiency (productivity and cost) and effectiveness (quality of care). There is little doubt that interest in ambulatory EMRs has increased over the past decade, yet only a small fraction of providers have adopted such systems. This slow rate of adoption suggests resistance among physicians remains strong. In 2003 , over one-half (53\%) of the healthcare executives participating in an annual survey conducted by Modern Healthcare and PricewaterhouseCoopers cited physician acceptance as a barrier (Morrissey, 2005). According to Hier (2002), physician "buy-in" has been limited which has delayed widespread adoption of EMRs. Kadas (2002) observed that a combination of social, market and economic factors have prevented acceptance of computer-based patient records (CPRs). Khoury (1997) suggested that the perceived value of EMRs was inadequate to motivate physicians to use them. Physician resistance to EMRs has been attributed to a variety of factors including, but not limited to: 1) well-publicized EMR failures; 2) limited computer literacy on the part of physicians; 3) concerns over productivity (i.e., fear that an EMR would slow physicians down); 4) patient satisfaction, and 5) unreliable technology (e.g., Hier, 2002; Hodge, 2002; Kadas, 2002; Khoury, 1997). Kadas (2002) suggests that acceptance has also been adversely impacted by the absence of "reputable independent third party" research substantiating benefits.

If physician acceptance or "buy-in" is in fact a critical success factor in widespread adoption of EMRs, it is appropriate and necessary that the perceptions of physicians concerning such applications be examined. Unfortunately, many of the articles on this topic to date have relied on anecdotal evidence and/or been authored or co-authored by individuals affiliated with EMR vendors. The few studies that have empirically examined this topic have focused on EMRs for specific medical specializations (e.g., family physicians, Loomis, Ries, Saywell, \& Thakker, 2002; cardiology, Linney, 2003), addressed primarily cost-benefit considerations (Wang et al, 2003), described single clinic case studies (e.g., Schmitt \& Wofford, 2002) or examined a single dimension of EMR utilization (e.g., electronic documentation, Sangster \& Hodge, 2003). A thorough understanding of physician perceptions concerning EMRs is a necessary prerequisite to both marketing and ultimately implementing such tools.

Assessing physician perceptions concerning EMRs is complicated by the fact that there is no precise definition of such applications (Handler, 2001). Attempting to define what constitutes an EMR is nearly impossible due to their rapid evolution and the variability across clinical settings/implementations. EMRs have evolved from simple clinical reporting systems to more sophisticated applications that can document patient encounters and in some cases provide decision support to clinicians. Clinics with similar profiles can adopt the same EMR product, yet their respective implementation can be dramatically different. These differences reflect disparate environmental factors including, technical infrastructure (e.g., networks, databases, processors and 
point-of-care devices), degree of product customization, alternative implementation strategies, including interfaces to both clinical and practice management applications, and clinician readiness. As Handler (2001) observed, both vendors and end users have created their own EMR definitions. While some definitional differences are subtle and would have minimal impact on physician perceptions, others are substantial and could significantly influence both acceptance and utilization.

Gartner, a recognized leader in IS research, recognized that EMRs are evolving and developed a classification scheme to assist end-users and vendors (Handler, 2001). Gartner's classification scheme characterized differences across EMRs in terms of generations. According to the Gartner report, EMRs (referred to as CPRs) will "gradually evolve" through five generations with each subsequent generation possessing increasingly sophisticated and integrated functionality. Gartner contrasted the generations on the basis of eight core functions: clinical documentation and data capture, clinical display, workflow, clinical decision support (CDSS), store, knowledge management, security and process and communication. Acknowledgment of generational differences is important as physician perceptions will be expected to evolve in a concomitant manner.

According to the Gartner classification scheme, first generation EMRs are simple systems for both capturing and displaying clinical data. In contrast, second generation EMRs are expected to assist with documentation at the point of care. Whereas first generation EMRs allowed for the inclusion of scanned documents and transcribed text, second generation applications are expected to provide for collection of limited discrete data during patient encounters. The presence of physician order entry (POE) and simplistic decision support such as drug-drug interactions and drug allergies further differentiate second generation systems. Third generation EMRs would reflect enhanced clinical documentation and data capture, including mainly discrete and structured documentation. This generation would also afford caregivers with more flexibility in representing clinical data and support more advanced clinical decision support. In the future, fourth and fifth generation applications are expected to incorporate additional decision support and expert system logic and knowledge management. A more detailed description of these EMR generations is beyond the scope of this paper (See Handler, 2001 for more detail).

In many cases, EMR definitions advanced by vendors and IS staffs responsible for implementation have reflected feasible functionality rather than addressing what is realistic and attainable given the resources and constraints in clinical settings. Miscommunication, misinformation and misinterpretation between vendors, clinic executives, IS leaders, and end-users (physicians and staff) has contributed to a myriad of problems in the marketing, selection, implementation and utilization of EMRs (Frabotta, 2002). The hype surrounding EMRs often created unrealistic expectations among physicians. The gap between expectations and EMR performance resulted in clinician dissatisfaction, unused technology and ultimately project failure and wasted capital expenditures (Handler, 2001). Such failures in the early and late ' 90 's provided support for EMR detractors seeking to delay application upgrades or initial adoptions. The aggressive marketing of EMR products, publicity of EMR successes and failures, lack of third party research regarding effectiveness and other factors have shaped physician perceptions over time. As these perceptions evolve, so does the need for ongoing research to understand the mindset of physicians concerning EMRs.

\section{Opportunities for Enhanced Physician-Patient Communication}

The maturing functionality afforded by each generation of EMR (as described by Handler, 2001) presents additional opportunities for enhancing quality of care. In some cases such systems will allow patients to provide medical histories once and have them available to multiple care providers. In other cases, EMRs will present findings that can be displayed graphically for the benefit of the patient. In other scenarios, templates, sometimes dynamically generated as a result of expert 
logic, will prompt physicians to collect additional medical history or order additional lab or diagnostic tests. The inclusion of rules or health reminders will allow EMRs to prompt care givers to remind patients that certain examinations or labs are recommended based on age, diagnosis and/or passage of time. In yet other cases, ready access to historical data will allow providers to address patient questions concerning everything from medication history to date of last exam, specialist reports to known allergies, and insurance coverage to disease management protocols. While a comprehensive review of all of the opportunities for quality of care is beyond the scope of this paper, specific examples described below illustrate the potential benefit.

\section{Reduced redundancy}

The limitation of paper charts maintained by individual physicians is evident during most office visits. At the start of most office visits patients are required to provide a litany of medical history (e.g., family medical history, allergies, medication, prior and existing condition, and surgeries). Due to medical charts rarely being shared by multiple providers this information is collected by each health care provider and often times collected on more than one occasion by the same provider. Depending upon the age or medical condition of the patient the amount of information required at the start of each office visit can be substantial. While some redundancy is intentional and desirable to validate medical conditions/data, patients not only resent having to recall this information on multiple occasions, but also unintentionally omit critical pieces of information. The implementation of an EMR would reduce the need for redundant data collection and allow care providers to quickly review the history and update the medical records where necessary. In those cases where the patient or family/guardian is unable to provide medical history having prior data available would be invaluable (e.g., elderly with Alzheimer, unconscious patients).

\section{Graphical display of results}

Over time patient charts contain considerable data regarding patient vitals (e.g., blood pressure, heart rate, and temperature), laboratory results (e.g., blood counts, blood sugars) and diagnostic tests (e.g., EKG). While discrete measures can be compared to norms, often times time trended data provides a more accurate view of a medical condition or concern. While physicians relying on paper charts can, and sometimes do, create simple charts it is impractical to create graphical displays for each clinical measure lending itself to a graphical representation. EMRs would allow physicians to easily generate and review graphical representations of one or more measures for a designated period of time. Graphical displays showing increasing blood pressure or blood sugar levels might alert care givers of an emerging problem or in the case of a recognized issue help reinforce the severity with the patient.

\section{Formulary decision making}

When determining what medication to prescribe a physician must consider both efficacy and cost. Prescribing medications that are beyond the economic means of patients is counter-productive, as is prescribing medications that are either not included in their insurance plan formulary or require a generic to qualify for a co-pay. Given the rapid increase in medication options and variation and rate of change in health care insurance benefits it is impractical, if not impossible for physicians to quickly assess the various options when using paper based systems. As a result, most patients do not learn the cost of medications nor the extent, if any, of insurance coverage until they present the prescription to a pharmacy. Patients, upon learning from the pharmacy that lower cost generics are available or that generics or other similar medications would be covered by insurance, frequently request that the physician prescribe an alternative medication. These requests are time consuming for the patient, provider and pharmacy. With an EMR, at the point of care (i.e., time of examination) a care provider can assess whether a medication is covered by a patient's insurance and discuss alternatives with them. EMRs can deliver this functionality by utilizing 
databases for both FDA approved medications and insurance plan formularies. Thus, in an EMR setting the physician can discuss medication options, including level of insurance coverage, during the examination. Further, once a medication is selected it is possible to transmit the prescription electronically to a pharmacy designated by the patient.

\section{Medication\Allergy\Problem documentation}

With complex medical conditions or instances where a patient sees multiple care providers it is difficult for both patients and physicians to recall medication, allergy and problem documentation. A single repository of such information combined with decision support could easily highlight unsafe interactions. Unsafe drug-drug or drug-allergy interactions could be identified as soon as the physician selects a medication on an electronic prescription pad (i.e., form). If postexam lab results suggest a previously prescribed medication is counter-indicated an EMR can automatically alert the physician and/or advise the patient to contact their physician. Further, if evidence based medicine (i.e., best practices) calls for a patient with a particular diagnosis to be examined every $n$ days, weeks or months an EMR can easily identify those patients overdue for a follow-up examination.

\section{Patient education}

A wide variety of patient education literature is available from medical associations and societies, pharmaceutical companies, government agencies and other organizations. For specialists that concentrate on a limited number of problems it may be practical to keep patient education handouts on file for distribution. However, as the scope of problems commonly treated increases so does the challenge of storing and retrieving paper-based patient education materials. For example, the myriad of medical conditions treated by primary care physicians makes it impractical to keep handouts on file for every aliment and treatment. EMRs that link diagnosis codes to related patient education literature will allow physicians to quickly and easily generate patient education material on demand, at the point of care. For physicians in high volume practices where multitasking is the norm, such material could be printed and presented for the patient to review while the physician treats another patient. Following treatment of the second patient, the physician could rejoin the first patient to discuss the information presented and answer questions. Providing specific and detailed patient education materials can improve patient compliance with care directives and reduce the number of post-visit telephone calls concerning patient education related matters.

While these examples are far from exhaustive, they serve to illustrate the potential for EMRs to improve quality of care. According to Frabotta (2002) quality-of-care has emerged as a focal point for most health care organizations as the traditional emphasis on return on investment has failed to drive widespread adoption. Proving the worth of EMRs to improve quality of care is critical as once clinicians recognize these opportunities and the opportunity costs of not changing, physician resistance will diminish.

\section{Research Methodology}

\section{Sample}

To examine physician perceptions related to ambulatory EMRs, a mail survey of 358 physicians affiliated with a large, multi-specialty clinic in the Midwest was conducted. The clinic is a physician-led, professionally managed group practice in an integrated health-care system. Questionnaires were mailed to physician homes in February 2003, with follow-up mailings to nonrespondent's homes ( 3 weeks later) and offices ( 5 weeks later). Preaddressed, postage-paid return envelopes were provided. Of $266(74 \%)$ responses, $5(1.4 \%)$ were excluded because half or more of the questions were unanswered. There were 261 (73\%) usable questionnaires. The high response 
rate and percentage of usable questionnaires was a result of reminders by administrators and physician-executives to participate in the research. These reminders were conveyed via email, weekly newsletters and staff meetings. The responses were entered into a spreadsheet and the data entry was verified for accuracy via manual verification; the data were imported into and analyzed using SPSS (Version 11.0.1).

\section{Questionnaire}

As part of an EMR readiness assessment the author designed a multi-section questionnaire based on previous EMR research focusing on critical success factors, physician acceptance/resistance and functionality. Individual sections of the questionnaire addressed: data requirements (inpatient and/or outpatient), use of existing systems, importance of EMR functions, anticipated utilization of EMR functions and general perceptions regarding the need and usefulness of an EMR. The questionnaire also invited respondents to share their general concerns regarding EMRs. Additional data, beyond the scope of the present study, was also collected.

\section{Findings}

\section{Respondent profile}

A profile of the respondents is presented in Table 1. These respondents could be broadly categorized into five physician groups: 1) Hospital Based (e.g., Radiology, Pathology, Hospitalists, Emergency Room); 2) Medical Specialties (e.g., Ophthalmology, Allergy, Rheumatology); 3) Primary Care (e.g., Family Medicine, Internal Medicine and Pediatrics); 4) Regional Providers (rural clinics and community hospital based physicians comprising Internal Medicine, Family Medicine/Obstetrics and General Surgery); and 5) Surgery. Consistent with national physician demographics the sample contains significantly more male $(211,81 \%)$ than female $(50,19 \%)$ respondents. Respondents ranged in age from 29.6 to 75.9 years with a mean of

\section{Data requirements}

Table 2 summarizes respondents requirements related to inpatient vs. out-patient data and prior experience accessing clinical data (e.g., labs and/or transcribed notes) online. A significantly greater proportion ( $93 \%$ vs $83 \%$ ) of physicians reported requiring access to outpatient versus inpatient data. While the majority of
Table 1: Respondent Profile

$\begin{array}{lcc}\begin{array}{l}\text { Gender } \\ \text {-- Male }\end{array} & 211 & (81 \%) \\ \text {-- Female } & 50 & (19 \%) \\ \text { Age (years) } & & \\ \text {-- Mean } & 46 & \\ \text {-- SD } & 8.7 & \\ \text {-- Range } & 29.6 \text { to } 75.9 \\ \text { Department } & & \\ \text {-- Hospital* } & 28 & (10.9 \%) \\ \text {-- Medical Specialties } & 52 & (20.3 \%) \\ \text {--Primary Care } & 64 & (25.0 \%) \\ \text {--Regional** } & 52 & (20.3 \%) \\ \text {--Surgery } & 62 & (24.2 \%) \\ \begin{array}{l}\text { * Includes Radiologists, Anesthesiologists, Pathologists and } \\ \quad \text { Hospitalists }\end{array} \\ \begin{array}{l}\text { ** Includes Family Medicine, OB/GYN and General Surgeons } \\ \text { affiliated with small clinics and/or community hospitals. }\end{array}\end{array}$

Table 2: Data Access Requirements

\begin{tabular}{lccc}
\hline Clinical Data Required: & Yes & No & $\begin{array}{c}\text { If "yes" } \\
\text { pct of } \\
\text { patients }\end{array}$ \\
\hline $\begin{array}{l}\text { Inpatient } \\
\text { Outpatient }\end{array}$ & $93 \%$ & $7 \%$ & $78 \%$ \\
$\begin{array}{l}\text { Currently Accessing data } \\
\text { online* }\end{array}$ & $83 \%$ & $17 \%$ & $45.8 \%$ \\
$\begin{array}{l}\text { *A limited function EMR was available for accessing lab results } \\
\text { and some clinical notes. }\end{array}$ & &
\end{tabular}


physicians expressed interest in accessing outpatient data electronically, the percentage of patients for whom this data was necessary ranged from $5 \%$ to $100 \%$ with a mean of $78 \%$. For physicians $(83 \%)$ requiring access to inpatient data the percentage of patients for whom accessed was needed ranged from $1 \%$ to $100 \%$ with a mean of $45.8 \%$. Less than one-half (43\%) of the respondents were presently accessing patient data online. It should be noted that this percentage may be misleading as a review of system access logs suggest far fewer physicians were logging on to access such information. This discrepancy is likely a result of physicians equating the review of reports printed by staff and placed in patient charts as accessing data online.

\section{Importance of EMR functions}

Table 3 summarizes the respondents' perceptions regarding the importance of specific EMR functions. Respondents were presented with a list of nineteen functions associated with what has been described as a Second Generation EMR (Handler, 2001). Respondents considered all of the EMR functions presented to be at least slightly important (mean $>=3.0$ ). The mean responses for the nineteen EMR functions ranged from 1.4 (Very Important to Quite Important) to 3.0 (Slightly Important). As noted in Table 3, the retrieval and display of clinical data was of greatest importance to respondents. Despite this interest in accessing clinical data, respondents rated a workflow inbox for office and/or hospital results as one of the least important functions (mean rating 2.8). Display of prescription alerts was considered slightly more important (mean 2.2) than the ability to prescribe online (mean 2.7). Structured documentation and physician order entry (POE) re-

Table 3: Importance of EMR Functions

\begin{tabular}{|c|c|c|}
\hline \multirow[b]{2}{*}{ Function } & \multicolumn{2}{|c|}{ Importance of Functior } \\
\hline & $\begin{array}{l}\text { Mean } \\
\text { Score* }\end{array}$ & Rank \\
\hline Display of Lab Results & 1.4 & 1 \\
\hline Display of Radiology Reports & 1.5 & 2 \\
\hline Display of Clinical Notes and Reports & 1.6 & 3 \\
\hline Display of Height, Weight and Allergies & 1.9 & 4 \\
\hline Nuclear Medicine & 1.9 & - \\
\hline Display of Radiology Images & 2.0 & 6 \\
\hline Entry/Display of Diagnoses and Medications & 2.0 & - \\
\hline Display of Other Ancillary Clinical Data & 2.2 & 8 \\
\hline $\begin{array}{l}\text { Prescription Alerts drug-drug, allergy and dose checking and } \\
\text { formulary management }\end{array}$ & 2.2 & - \\
\hline Display of Demographics & 2.5 & 10 \\
\hline Time trended Clinical Data Display & 2.5 & - \\
\hline Structured Documentation (Templates) & 2.5 & - \\
\hline Physician Order Entry (tests and medication orders) & 2.6 & 13 \\
\hline Prescription Writing & 2.7 & 14 \\
\hline Workflow Inbox for office and/or hospital results & 2.8 & 15 \\
\hline Decision Support (guidelines, expert logic, reminders/alerts) & 2.9 & 16 \\
\hline Preventative Health Reminders & 3.0 & 17 \\
\hline Medical Management Reporting - notification by diagnoses & 3.0 & - \\
\hline Medical Management Reporting - disease management reporting & 3.0 & - \\
\hline
\end{tabular}


ceived mean scores of 2.5 and 2.6, respectively. Despite the promise of EMRs to offer health reminders and decision support at the point of care these functions along with medical management reporting were rated the least important by respondents.

\section{Expected utilization}

Table 4 summarizes respondents anticipated utilization of each EMR function in their practice. Mean responses for these items suggest 15 of the 19 EMR functions would be used at least weekly. Not surprisingly, anticipated utilization of functions is highly correlated with perceived importance. Only preventative health reminders, decision support capabilities, and medical management reporting generated mean responses suggesting less than weekly usage. For 13 of the 19 functions at least one-half of the respondents anticipated daily use for some, if not all, of their patients. Consistent with the importance ratings respondents' anticipated greater utilization of functions related to the retrieval and display of clinical data.

Table 4: Anticipated Utilization of EMR Functions

\begin{tabular}{|c|c|c|}
\hline \multirow[b]{2}{*}{ Function } & \multicolumn{2}{|c|}{ Anticipated Utilization } \\
\hline & $\begin{array}{l}\text { Mean } \\
\text { Score* }\end{array}$ & Rank \\
\hline Display of Lab Results & 1.7 & 1 \\
\hline Display of Clinical Notes and Reports & 1.7 & - \\
\hline Display of Radiology Reports & 1.8 & 3 \\
\hline Display of Height, Weight and Allergies & 2.0 & 4 \\
\hline Display of Radiology Images & 2.2 & 5 \\
\hline Entry/Display of Diagnoses and Medications & 2.2 & - \\
\hline Display of Other Ancillary Clinical Data & 2.3 & 7 \\
\hline $\begin{array}{l}\text { Prescription Alerts drug-drug, allergy and dose checking and } \\
\text { formulary management }\end{array}$ & 2.3 & - \\
\hline Display of Demographics & 2.4 & 9 \\
\hline Nuclear Medicine & 2.5 & 10 \\
\hline Prescription Writing & 2.5 & - \\
\hline Time trended Clinical Data & 2.7 & 12 \\
\hline Workflow Inbox for office and/or hospital results & 2.7 & - \\
\hline Physician Order Entry (tests and medication orders) & 2.7 & 13 \\
\hline Display Structured Documentation (Templates) & 2.9 & 15 \\
\hline Decision Support (guidelines, expert logic, reminders/alerts) & 3.1 & 16 \\
\hline Preventative Health Reminders & 3.2 & 17 \\
\hline Medical Management Reporting - notification by diagnoses & 3.4 & 18 \\
\hline Medical Management Reporting - disease management reporting & 3.6 & 19 \\
\hline
\end{tabular}

\section{Physician attitudes and beliefs}

Table 5 summarizes respondents' general attitudes and beliefs regarding EMRs, including familiarity with functions and benefits, impacts, usage/training and overall value and need for adoption. Both the mean rating for each item and the percentages "agreeing" and "disagreeing" are presented. These percentages were calculated by collapsing a six point Likert scale from (1) Strongly 
Agree to (6) Strongly Disagree to create a dichotomous variable, "agree" and "disagree." A majority (56 percent) of the respondents expressed doubt that physicians were familiar with EMR functions and benefits. Over 80 percent of the respondents felt an EMR would improve quality of care, while 76.6 percent expected the impact on quality of practice (i.e., work life) to be positive. Slightly fewer of the respondents $(60.2 \%)$ expect an EMR will increase practice productivity. Nearly three-quarters $(71.5 \%)$ of the respondents felt EMR usage would have to be mandated. Nearly one-third (31.7\%) of the respondents expressed doubt that physicians would devote the time required for EMR training. The majority of respondents felt that benefits of an EMR outweigh the costs (72\%) and that an EMR should be implemented (79.6\%).

Table 5: Attitudes and Beliefs Regarding EMRs

\begin{tabular}{|c|c|c|c|}
\hline & $\underline{\text { Mean* }}$ & $\begin{array}{c}\text { Pet. } \\
\text { Agreeing }\end{array}$ & $\begin{array}{c}\text { Pct. } \\
\text { Disagreeing }\end{array}$ \\
\hline \multicolumn{4}{|l|}{ Familiarity } \\
\hline $\begin{array}{l}\text { Physicians are familiar with EMR functions and } \\
\text { benefits }\end{array}$ & 3.74 & 43.8 & 56.2 \\
\hline \multicolumn{4}{|l|}{ Impact } \\
\hline$\overline{\text { EMRs improve quality of care and reduce errors }}$ & 2.42 & 82.7 & 17.3 \\
\hline EMRs improve quality of practice (i.e., work life) & 2.66 & 76.6 & 23.4 \\
\hline $\begin{array}{l}\text { EMRs increase practice productivity (i.e., patients per } \\
\text { day) }\end{array}$ & 3.22 & 60.2 & 39.8 \\
\hline \multicolumn{4}{|l|}{ AcceptancelTraining } \\
\hline EMR usage will have to be mandated & 2.77 & 71.5 & 28.5 \\
\hline $\begin{array}{l}\text { Physicians will devote the time required for EMR } \\
\text { training }\end{array}$ & 3.08 & 68.3 & 31.7 \\
\hline \multicolumn{4}{|l|}{ General } \\
\hline EMR benefits outweigh the costs & 2.72 & 72.0 & 28.0 \\
\hline An EMR system should be implemented & 2.49 & 79.6 & 20.4 \\
\hline
\end{tabular}

Respondents were provided with additional space to express general concerns regarding EMRs. As might be expected, the open-ended section generated a wide range of issues including: learning curve, impact on productivity, response time, cost, security, patient acceptance, privacy, complexity, training needs and physician resistance. It is noteworthy only a small number of comments addressed patient acceptance (9) and physician resistance (15). Physicians concerned about patient acceptance commonly noted that they (physicians) would spend more time interacting with the computer than the patient. In these cases it was clear that the respondents did not view the EMR as an opportunity to enhance or improve physician-patient communication. Comments regarding physician resistance generally were in regards to other physicians being opposed to implementation of an EMR. A small number of respondents were concerned about security and/or privacy, however, that number was much smaller than one might expect given the attention that the Health Insurance Portability and Accountability Act (HIPPA) has garnered in recent years.

\section{Discussion}

Differences noted in access requirements for inpatient and outpatient clinical data likely reflect differences in medical practice. As stated earlier, this research was conducted in a clinic that was part of an integrated health-care system. While many of the respondents cared for both inpatients as well as outpatients, some physicians dealt strictly with outpatients while others restricted their 
practice to inpatients. In retrospect, categorizing patients as "inpatient" and "outpatient" may have failed to address a growing phenomenon of "Outpatient Surgery." While the patient is technically an "outpatient," the data associated with outpatient surgery is often viewed as inpatient data as surgery centers are frequently operated by hospitals.

The relative importance of clinical data retrieval and display vis-à-vis other EMR functions (see Table 3) is not surprising given the respondents familiarity with and use of paper-based medical records. Physicians who have relied on paper charts to provide patient vitals, lab results, radiology and other clinical reports, and other ancillary clinical data would project this dependence to an EMR and view these functions as critically important. The finding that prescription alerts are considered more important than electronic prescription writing may reflect a growing awareness of the potential for EMRs to dramatically reduce adverse drug reactions (ADRs). Structured documentation (templates) and physician order entry (POE) may have been rated lower in importance as physicians have developed strategies and methods for quickly completing these common tasks. In the case of documentation, physicians frequently utilize dictation with abbreviations and common entries that are transcribed into "richer" notes. Orders, typically in well defined sets, are easily and quickly issued verbally to clinical staff for execution and documentation.

The lower importance rating for a digital workflow inbox was somewhat surprising given the daily volume of office and hospital results, orders and communication requiring physicians' attention. Subsequent dialogue with physicians confirmed that most physicians did not fully appreciate this particular functionality nor the benefits it would afford clinical staff. The lower importance ranking for Decision Support (guidelines, expert logic, and reminders/alerts) may be attributed to the misconception that such functionality devalues the medical knowledge and expertise of physicians. Physicians trained to be knowledgeable and decisive struggle with the concept of decision support and this may have resulted in some respondents minimizing the importance of this functionality. This may be equally true in the case of preventative health reminders. Physicians that have historically relied on memory and personal judgment to address preventative health issues may be hesitant to rate preventative health reminders higher in importance as it could be construed as an indictment of their current practice. The fact that medical management reporting, notification by diagnoses and disease management reporting, scored the lowest in importance is not surprising given the paucity of such reporting at present. Such reporting is time consuming and expensive with paper charts and physicians may not appreciate the potential for such reports to improve quality of care.

The anticipated utilization of EMR functions was found to be highly correlated with the perceived importance. While select functions were ranked a few positions higher/lower it was evident that respondents equate importance with utilization. Given the professed lack of familiarity with EMR functions on the part of respondents it is plausible to suggest that experience with these functions will likely change perceptions regarding importance and utilization rates.

The respondents' general attitudes and beliefs (See Table 5) suggest overall support for EMRs. Over three-quarters of the respondents agreed that EMRs would improve both quality of care and quality of practice (i.e., work life). To a lesser extent, respondents believe that practice productivity would increase with an EMR. This later perception may reflect in part physician experiences with early clinical systems that often offered little in the way of time savings (Treister, 1998). The finding that nearly three-quarters of the physicians felt usage would have to be mandated is consistent with Hier (2002) who recommended mandating EMR utilization. The findings suggest a significant percentage of physicians may be unwilling to devote sufficient time to EMR training. Given physician concerns regarding productivity it is critical that they be motivated (e.g., incentives) to participate in training. Since the majority of physicians currently believe EMR benefits outweigh costs, it comes as no surprise that nearly 80 percent feel an EMR system should be implemented. 


\section{Limitations and Future Research}

There are limitations to all survey based research, and it is important to note those which may affect findings from this study. First, one can question whether the sample is representative of all clinic settings and medical specialties. While most medical specialties were represented, the sample was limited to physicians affiliated with a single, large multi-specialty clinic. While the physicians were employed by the clinic, it is important to note that they operated under the oversight of a physician elected board and the vast majority had their compensation tied directly to productivity. Further, one might question whether past efforts by this clinic to adopt an EMR, including several rounds of EMR demos over a ten year span, may have biased the results of this study. Finally, other variables such as age, gender, previous experience with clinical information systems, etc. may have impacted the results. The fact that over one-half $(56 \%)$ of the participating physicians expressed concern that physicians in general were unfamiliar with EMR functions and benefit suggests a lack of prior knowledge may have influenced responses. The extent to which the findings were affected by these limitations is unknown. These issues and others noted below should be addressed by further research.

It is clear that additional understanding of physician perceptions will aid in the development and marketing of functional, "user-friendly" EMR applications in the ambulatory marketspace. The present study broadly examined physician perceptions in a large multi-specialty clinic setting. To more accurately assess physician perceptions of EMRs, future research should address other clinical settings (e.g., small clinics, managed care clinics, profit vs. nonprofit), examine differences across medical specialties, and assess the impact of varied demographic factors. Looking beyond the adoption decision, researchers should also begin investigations to assess the impact of alternative training models, implementation strategies (e.g., phased vs. "big bang") and point of care devices on EMR adoptions, utilization and outcomes. Such research would provide valuable insight concerning physician perceptions and acceptance of EMR applications and associated benefits.

Future studies are also needed to address patient perceptions. With increased emphasis being placed on patient satisfaction, physicians and healthcare organizations need to develop strategies to allay patient concerns and fears related to the introduction of EMRs. And, if healthcare providers are to successfully highlight EMR capabilities when marketing their services they must first understand what consumers perceive to be the most important functions.

\section{Summary}

While there are many barriers to widespread adoption of EMRs those associated with physicians are not insurmountable. On the contrary, by systematically examining physician perceptions related to EMRs both vendors and healthcare organizations can accelerate physician acceptance and ultimately the rate of adoption and utilization. Understanding physician perceptions will allow for the development of targeted education to demonstrate the advantages of EMRs and further improve physician perceptions of EMRs (Loomis et al., 2002). Likewise, fears regarding lack of patient acceptance of EMRs can be addressed by touting the opportunities for enhanced physician-patient communication. Physician resistance to ambulatory EMRs is not unlike that experienced in other software markets over the past several decades. As in those marketspaces, the combination of increasingly sophisticated functionality, including improved user-interfaces, increasing numbers of successful implementations, growing consumer expectations for information only accessible via computer-based systems, and increased physician (i.e., end-user) awareness of functionality and benefits will ultimately lead to widespread adoption. 


\section{References}

Frabotta, D. (2002, June). EMRs require clinician approvals to reach significant adoption levels. Managed Healthcare Executive, 48-50.

Handler, T. (2001). CPR generations: an update (Report No: TU-12-9718). GartnerGroup.

Hier, D. B. (2002, October). Physician buy-in for an EMR. Healthcareinformatics. Retrieved November 18,2004 , from http://www.healthcare-informatics.com/issues/2002/10 02/commentary.htm

Hodge, R. (2002, January-February). Myths and realities of electronic medical records, The Physician Executive, 14-19.

Kadas, R. M. (2002, February). The computer-based patient record is on its way. Healthcareinformatics. Retrieved November 18, 2004, from http://www.healthcareinformatics.com/issues/2002/02_02/leading.htm

Linney, B. (2003, May-June). Cardiology practice proves that electronic medical records do raise revenue. The Physician Executive, 34-36.

Loomis, G, A., Ries, J. S., Saywell, R. M. \& Thakker, N. R. (2002). If electronic medical records are so great, why aren't family physicians using them? The Journal of Family Practice, 51(7), 636-641.

Morrissey, J. (2005). This time they really mean it. Modern Healthcare, 35(7), 42-48.

Renner, K. (1996, October). Cost-justifying electronic medical records. Healthcare Financial Management, 63-70.

Sangster, W. M. \& Hodge, R. H. (2003, March April) Electronic documentation vs. dictation: How do they compare? The Physician Executive, 26-29.

Schmitt, K. F. \& Wofford, D. A. (2002, January). Financial analysis projects clear returns from electronic medical records. Healthcare Financial Management, 52-57.

Treister, N. W. (1998). Physician acceptance of new medical information systems: The field of dreams. Physician Executive, 24(3), 20-24.

Versel, N. (2002). Stalking the elusive 'Killer App'. Modern Physician, 6(5). Retrieved March 10, 2004, from http://modernphysician.com/articles:cms?articleID $=148$

Wang, S. J., Middleton, B., Prosser, L. A., Bardon, C. G., Spurr, C. D., Carchidi, P. J., Kittler, A. F., Goldszer, R. C., Fairchild, D. G., Sussman, A. J., Kuperman, G. J. \& Bates, D. W. (2003). A costbenefit analysis of electronic medical records. The American Journal of Medicine, 114(5), 397-403.

\section{Biography}

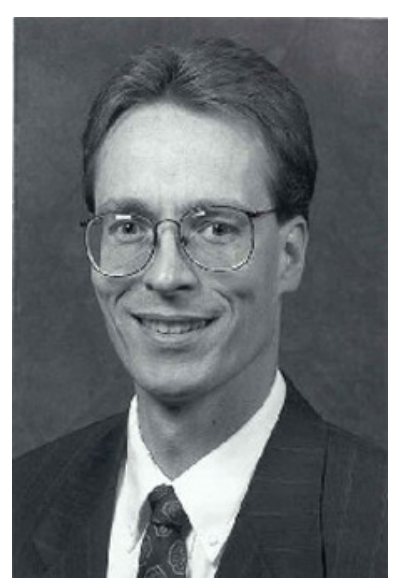

David B. Meinert is Professor and Director of the MS CIS Program at Southwest Missouri State University. Dr. Meinert received his doctorate in management information systems from the University of Mississippi in 1990. His professional background includes software development, systems integration and project management. Dr. Meinert has published in a number of journals, including Journal of Applied Business Research, Journal of Computer Information Systems, Information Strategy: The Executive's Journal, Information Resource Management Journal, End User Computing Management, Focus on Change Management, Journal of Marketing Management, and Journal of Marketing Theory and Practice. 